\title{
Acknowledgement to Referees for Skin Appendage Disorders 2019
}

\section{Skin \\ Appendage Disorders}

Josette Andre, Brussels, Belgium

Daniel Asz Sigall, Mexico City, Mexico

Robert Baran, Cannes, France

Richert Bertrand, Brussels, Belgium

Vincenzo Bettoli, Ferrara, Italy

Nisha Suyien Chandran, Singapore, Singapore

George Cotsarelis, Philadelphia, PA, USA

Ralph Daniel, Jackson, MS, USA

David de Berker, Bristol, United Kingdom

Desmond Tobin, Dublin, Ireland

Rachita Dhurat, Mumbai, India

Nilton Di Chiacchio, São Paulo, Brazil

Ncoza Dlova, Durban, South Africa

Isabella Doche, São Paulo, Brazil

Judith Dominguez-Cherit, Mexico City,

Mexico

Aline Donati, São Paulo, Brazil

Andrei Doroshkevich, Russia

Zoe Diana Draelos, High Point, NC, USA

Boni Elewski, Birmingham, AL, USA

Gorana Epstein, Miami, FL, USA

Gabriella Fabbrocini, Naples, Italy

Harold Farber, Philadelphia, PA, USA

Steven Feldman, Winston-Salem, NC, USA

Lynne Goldberg, Boston, MA, USA

Stamatios Gregoriou, Athens, Greece

Ramon Grimalt, Sant Cugat del Valls, Spain

Chander Grover, Delhi, India

Aditya K. Gupta, London, ON, Canada

The Editors extend their gratitude and appreciation to the following reviewers whose comments and criticisms ensure the quality of the papers published in this journal.

Roberto Arenas Guzmán, Tlalpan, Mexico

Daniela Araucaria Guzmán-Sánchez, Guadalajara, Jalisco, Mexico

Eckart Haneke, Freiburg, Germany

Molly Hinshaw, Madison, WI, USA

Dimitrios Ioannides, Thessaloniki, Greece

Matilde Iorizzo, Bellinzona, Switzerland

Nathaniel Jellinek, East Greenwich, RI, USA

Ana Kaminsky, Buenos Aires, Argentina

Alexandros Katoulis, Athens, Greece

Yanna Kelly, São Paulo, Brazil

Thomas Knackstedt, Cleveland, OH, USA

Athanassios Kolivras, Brussels, Belgium

Francesco Lacarrubba, Catania, Italy

Michelangelo La Placa, Bologna, Italy

André Lencastre, Lisboa, Portugal

Shari Lipner, New York, NY, USA

María Abril Martinez-Velasco, Ciudad de México, Mexico

Amy J. McMichael, Winston-Salem, NC, USA

Natasha Mesinkovska, Irvine, CA, USA

Andrew G. Messenger, Sheffield, United Kingdom

Giuseppe Micali, Catania, Italy

Paradi Mirmirani, Vallejo, CA, USA

Mariya Miteva, Miami, FL, USA

Oscar M. Moreno-Arrones, Madrid, Spain

Dedee Murrell, Sydney, NSW, Australia

Yuliya Ovcharenko, Kharkiv, Ukraine

Suchismita Paul, Boston, MA, USA
Bianca Maria Piraccini, Bologna, Italy Rodrigo Pirmez, Rio de Janeiro, Brazil Adriana Rakowska, Warsaw, Poland Alfredo Rebora, Genova, Italy Phoebe Rich, Portland, OR, USA Bertrand Richert, Brussels, Belgium Bruno de Oliveira Rocha, Salvador, Brazil Ricardo Romiti, São Paulo, Brazil Adam I. Rubin, Philadelphia, PA, USA Lidia Rudnicka, Warsaw, Poland David Saceda-Corralo, Madrid, Spain Richard K. Scher, New York, NY, USA Jerry Shapiro, New York, NY, USA Avner Shemer, Tel Hashomer, Tel-Aviv, Israel

Rodney Sinclair, Melbourne, VIC, Australia

Sidharth Sonthalia, Gurugram, India

Leonardo Spagnol Abraham, Brasília, Brazil

Michela Starace, Bologna, Italy

Kumar Sukhdeo, New York, NY, USA

Chinmanat Tangjaturonrusamee,

Bangkok, Thailand

Curtis Thompson, Portland, OR, USA

Ralph Trüeb, Wallisellen, Switzerland

Sergio Vañó-Galván, Madrid, Spain

Norma Vazquez, Monterrey, Mexico

Colombina Vincenzi, Bologna, Italy

Uwe Wollina, Dresden, Germany

Martin Zaiac, Miami Beach, FL, USA

Abraham Zlotogorski, Jerusalem, Israel 